\title{
Editorial for the topical issue on the Continuous Time Random Walk ${ }^{\star}$
}

\author{
Ryszard Kutner ${ }^{1, a}$ and Jaume Masoliver ${ }^{2}$ \\ ${ }^{1}$ University of Warsaw, Institute of Experimental Physics, Hoza Str. 69, 00681 Warsaw, Poland \\ 2 Department of Condensed Matter Physics and Complex Systems Institute (UBICS), University of Barcelona, \\ Catalonia, Spain
}

Received 27 July 2017

Published online 19 February 2018 - (c) EDP Sciences, Società Italiana di Fisica, Springer-Verlag 2018

The purpose of this volume is to commemorate the 50th anniversary of the introduction of the Continuous Time Random Walk (CTRW) by Elliott W. Montroll (19161983) and George H. Weiss (1930-2017). As often occurs, the publication of the paper in 1965 stirred up very little attention, if any, until in the early seventies with the works of Harvey Scher and collaborators which applied the CTRW technique to what has been latter called anomalous diffusion (see the article by Harvey Scher appearing in this volume [1]). The article by Shlesinger [2] presents a complete introduction on the history and antecedents of the CTRW. After this modest beginning, the importance of the formalism soared with more and more works generalizing and applying the CTRW with the number of cites climbing to several tens of thousands. In this regard, see our introductory article to this topical issue [3].

The truth is that the CTRW constitutes an extremely powerful albeit relatively simple formalism to approach and eventually solve a countless number of problems in many areas of physical, natural and even socio-economic sciences.

As its name suggests the CTRW generalizes simple random walk models. Although the term "random walk" was coined by Pearson in 1905, the formalism had been formulated in the 17th century in the context of gambling games such as the probability of ruin after betting $n$ times in a coin tossing game. In this case, the sum of gains minus losses in $n$ trials is equal to the state of a player's fortune. Let us thus note that the ordinary random walk is based on the assumption that changes are made at equal time intervals. However this is a first approximation in the study of many natural and socio-economic phenomena. The CTRW relaxes this restriction since it assumes that time intervals between consecutive changes are not constant but random. This simple concept became the basis of the second youth of the field of random walk.

In fact, in the CTRW there are two sources of randomness: one coming from the waiting times and another

\footnotetext{
^ Contribution to the Topical Issue "Continuous Time Random Walk Still Trendy: Fifty-year History, Current State and Outlook", edited by Ryszard Kutner and Jaume Masoliver.

a e-mail: ryszard.kutner@fuw.edu.pl
}

from the amplitudes of random jumps. Thus, in one simple picture of the CTRW, a walker staying at $\mathbf{r}_{0}$ makes initially a random jump to $\mathbf{r}_{0}+\mathbf{r}$, then waits there during a random time interval $t$ and randomly jumps to a new position, waits there another (random) interval of time and makes another (random) jump, and so on. The walk is determined by two probability density functions, $\psi(t)$ for the waiting time intervals and $h(\mathbf{r})$ for position jumps. In one of the simplest versions waiting times and jumps are assumed to be independent of each other (though this is not the case of processes such as continuous-time Lévy flights where jumps and waiting times are correlated).

The probability density function, $p(\mathbf{r}, t)$, of the walker to be at $\mathbf{r}$ at time $t$, assuming that initially was at $\mathbf{r}_{0}=0$, is determined by $\psi(t)$ and $h(\mathbf{r})$ by means of the celebrated Montroll-Weiss equation for the joint FourierLaplace transform $\hat{\tilde{p}}(\boldsymbol{\omega}, s)$ of the probability density of the walker:

$$
\hat{\tilde{p}}(\omega, s)=\frac{[1-\hat{\psi}(s)] / s}{1-\hat{\psi}(s) \tilde{h}(\boldsymbol{\omega})},
$$

where $\hat{\psi}(s)$ and $\tilde{h}(\boldsymbol{\omega})$ are respectively the Laplace and Fourier transforms of waiting-time and jump densities. This formula is the icon of the CTRW.

As mentioned above, the CTRW essentially differs from the ordinary random walk in considering random waiting times and random jump sizes. The overall walk can be handled even if these times and jumps have no finite moments which permits an unified treatment of both fractional (i.e., fractal) and non-fractional random motion. Thus in the "fluid limit" - that is to say, large times and distances - one assumes, as $s \rightarrow 0$ and $|\boldsymbol{\omega}| \rightarrow 0$, that

$$
\hat{\psi}(s)=1-(T s)^{\alpha} \cdots, \quad \tilde{h}(\boldsymbol{\omega})=1-(L|\boldsymbol{\omega}|)^{2 \gamma} \cdots,
$$

where $0<\alpha \leq 1,0<\gamma \leq 1$ and $T$ and $L$ are positive constant parameters, $T$ sets a characteristic time and $L$ a characteristic length. When $\alpha=\gamma=1$, substituting these expansions into the Montroll-Weiss equation results in the usual Gaussian distribution of the Brownian motion. In this case there is a finite mean waiting time given by $T$ and a finite jump variance given by $L^{2}$. 
For fractional values of the exponents $\alpha$ and $\gamma$ one ends up with a fractal probability distribution of the Lévy type for which there is neither finite mean waiting time nor finite jump variance. In this way the CTRW became a foundation of anomalous (dispersive, non-Gaussian) transport which opened a most fashionable field in modern statistical mechanics and condensed and soft-matter physics that goes beyond the traditional Boltzmann-Gibbs statistical mechanics.

During the half-century elapsed since its introduction, theoretical developments and applications of the CTRW have been greatly expanded and cover numerous situations with countless applications and the work continues, as the reader can see in our introductory article to this topical issue. The volume contains a collection of papers on various aspects of the CTRW with a careful selection between review and novel research articles.

Unfortunately the originators of the CTRW no longer are among us. Elliott Montroll died on January 1983 and George Weiss on February 2017 while this commemorative volume was in progress. We dedicate this topical issue to the memory of these outstanding scientists.

\section{References}

1. H. Scher, Eur. Phys. J. B 90, 252 (2017)

2. M.F. Shlesinger, Eur. Phys. J. B 90, 93 (2017)

3. R. Kutner, J. Masoliver, Eur. Phys. J. B 90, 50 (2017) 